\title{
Discovery of millihertz quasi-periodic oscillations in the X-ray binary EXO 0748-676
}

\author{
G. C. Mancuso, ${ }^{1,2,3 \star}$ D. Altamirano, ${ }^{3 \star}$ F. García ${ }^{\oplus},{ }^{1,4}$ M. Lyu ${ }^{\odot},{ }^{5}$ M. Méndez, ${ }^{6}$ \\ J. A. Combi, ${ }^{1,2}$ M. Díaz-Trigo ${ }^{7}$ and J. J. M. in't Zand ${ }^{8}$ \\ ${ }^{1}$ Instituto Argentino de Radioastronomía (CCT-La Plata, CONICET; CICPBA), C.C. No. 5, 1894 Villa Elisa, Argentina \\ ${ }^{2}$ Facultad de Ciencias Astronómicas y Geofísicas, Universidad Nacional de La Plata, Paseo del Bosque s/n, 1900 La Plata, Argentina \\ ${ }^{3}$ Physics \& Astronomy, University of Southampton, Southampton, Hampshire SO17 1BJ, UK \\ ${ }^{4}$ Laboratoire AIM, CEA, CNRS, Université Paris-Saclay, Université Paris Diderot, Sorbonne Paris Cité, F-91191 Gif-sur-Yvette, France \\ ${ }^{5}$ Department of Physics, Xiangtan University, Xiangtan, Hunan 411105, China \\ ${ }^{6}$ Kapteyn Astronomical Institute, University of Groningen, PO BOX 800, NL-9700 AV Groningen, the Netherlands \\ ${ }^{7}$ ESO, Karl-Schwarzschild-Strasse 2, D-85748 Garching bei München, Germany \\ ${ }^{8}$ SRON Netherlands Institute for Space Research, Sorbonnelaan 2, NL-3584 CA Utrecht, the Netherlands
}

Accepted 2019 April 22. Received 2019 April 22; in original form 2019 February 25

\begin{abstract}
We report the discovery of millihertz quasi-periodic oscillations ( $\mathrm{mHz}$ QPOs) from the bursting, high-inclination atoll neutron star low-mass X-ray binary (NS LMXB) EXO 0748-676 with the Rossi X-ray Time Explorer (RXTE). This class of QPO, originally discovered in three NS LMXBs, has been interpreted as a consequence of a special mode of nuclear burning on the NS surface. Using all the RXTE archival observations of the source, we detected significant $(>3 \sigma) \mathrm{mHz}$ QPOs in 11 observations. The frequency of the oscillations was between $\sim 5$ and $\sim 13 \mathrm{mHz}$. We also found a decrease of the QPO frequency with time in two occasions; in one of these the oscillations disappeared with the onset of an X-ray burst, similar to what was reported in other sources. Our analysis of the X-ray colours revealed that EXO 0748-676 was in a soft spectral state when it exhibited the QPOs. This makes EXO 0748-676 the sixth source with $\mathrm{mHz}$ oscillations associated with marginally stable burning, and the second one that shows a systematic frequency drift. Our results suggest that the mechanism that produces the drift might always be present if the $\mathrm{mHz}$ QPOs are observed in the so-called intermediate state.
\end{abstract}

Key words: accretion, accretion discs - stars: individual: EXO 0748-676-stars: neutron$\mathrm{X}$-rays: binaries.

\section{INTRODUCTION}

Low-mass X-ray binaries (LMXBs) are some of the brightest X-ray sources in the Galaxy. These systems consist of a compact object (a neutron star or a black hole) and a 'normal' (generally a mainsequence $\lesssim 1 \mathrm{M}_{\odot}$ ) companion star. These LMXBs are powered by mass accretion, from the donor star to the compact object, through Roche lobe overflow. Because of the conservation of angular momentum, this material does not fall directly on to the compact star but forms an accretion disc around it (Pringle \& Rees 1972).

Neutron star (NS) LMXBs are classified into two main groups based on their timing and spectral properties, the $\mathrm{Z}$ and atoll sources, the latter being fainter than the former (Hasinger \& van

^E-mail: gmancuso@iar.unlp.edu.ar (GCM); d.altamirano@soton.ac.uk (DA) der Klis 1989). In turn, the atoll sources are divided in three main spectral states: hard (or extreme island), soft (or banana), and intermediate (or island) states. NSs in LMXBs are usually thought to be weakly magnetized $\left(\mathrm{B}<10^{10} \mathrm{G}\right.$; e.g. Psaltis \& Chakrabarty 1999 and references therein). Many of these systems show X-ray bursts, a sudden and very rapid increase in the X-ray flux due to a thermonuclear runaway, followed by an exponential decay due to the cooling of the NS (see e.g. Strohmayer \& Bildsten 2006). Most of the NS LMXBs show X-ray variability in the form of relatively sharp $\mathrm{mHz}$ to $\mathrm{kHz}$ quasi-periodic oscillations (QPOs), and broad-band noise at $<100 \mathrm{~Hz}$ (see e.g. review by van der Klis 2006). Typical well-defined classes of QPOs are, among others, the so-called (i) kHz QPOs, which generally appear in pairs in the 300 $1300 \mathrm{~Hz}$ range (see e.g. van der Klis 2006), (ii) hectohertz $(\mathrm{hHz})$ QPOs, with frequencies in the range of $\sim 100-300 \mathrm{~Hz}$ (e.g. van Straaten, van der Klis \& Méndez 2003, Altamirano et al. 2008b), (iii) low-frequency QPOs, which have frequencies in the $0.01-50 \mathrm{~Hz}$ 
range (e.g. Jonker, van der Klis \& Wijnands 1999; Jonker, Méndez \& van der Klis 2000; Homan, Wijnands \& van den Berg 2003; van Straaten et al. 2003; Wijnands 2004) depending on the source state and luminosity, and (iv) $\mathrm{mHz}$ QPOs, observed at frequencies between 6 and $15 \mathrm{mHz}$ in five NS LMXBs (4U 1636-53, 4U 1608-52, and Aql X-1, Revnivtsev et al. 2001; 4U 1323-619, Strohmayer \& Smith 2011; and GS 1826-238, Strohmayer et al. 2018), ${ }^{1}$ all of which are reported or candidate atoll sources.

The $\mathrm{mHz}$ QPOs have properties that set them apart from the other types of QPOs: $\mathrm{mHz}$ QPOs occur only within a narrow range of X-ray luminosities $\left(L_{2-20 \mathrm{keV}} \simeq(0.5-1.5) \times 10^{37} \mathrm{erg} \mathrm{s}^{-1}\right)$, their fractional rms amplitude increases towards low energies, and the QPOs disappear at the onset of a thermonuclear (type I) X-ray burst (Revnivtsev et al. 2001, Altamirano et al. 2008a). Revnivtsev et al. (2001) suggested that the $\mathrm{mHz}$ QPOs could correspond to a special mode of nuclear burning on the NS surface, although they could not rule out an explanation due to instabilities in the accretion disc. The results of Yu \& van der Klis (2002) further supported the thermonuclear interpretation: they found that in the atoll source $4 \mathrm{U}$ $1608-52$ the frequency of the $\mathrm{kHz}$ QPO was anticorrelated with the $2-5 \mathrm{keV}$ X-ray count rate variations during the $\mathrm{mHz}$ oscillation. This anticorrelation suggests that the inner edge of the accretion disc increases due to the additional flux produced in each $\mathrm{mHz}$ QPO cycle on the NS surface. This is opposite to the positive correlation observed on longer time-scales between the $\mathrm{kHz}$ QPO frequency and the X-ray count rate, consistent with the radius of the inner disc edge decreasing as the accretion rate increases (see e.g. Méndez et al. 1999).

Altamirano et al. (2008a) found a systematical decrease of the $\mathrm{mHz} \mathrm{QPO}$ frequency with time in the NS LMXB 4U 1636-53. Once the frequency dropped below $9 \mathrm{mHz}$, a bright type I X-ray burst occurred and the oscillations disappeared. This drifting behaviour was only observed when $4 \mathrm{U} 1636-53$ was close to the transition between the hard and soft states. Altamirano et al. (2008a) showed that the $\mathrm{mHz}$ QPO frequency can be used to predict the occurrence of a type I burst, strengthening the interpretation that the $\mathrm{mHz}$ QPO phenomenon is intimately related to nuclear burning on the NS surface.

Additional work of Lyu, Méndez \& Altamirano (2014) and Lyu et al. $(2015,2016)$ investigated the properties of the $\mathrm{mHz}$ QPOs in 4U 1636-53. Using RXTE and XMM-Newton observations, the authors found that the $\mathrm{mHz}$ QPO frequency and the temperature of the NS surface were not significantly correlated. This finding is not consistent with theory, which predicts an anticorrelation between those parameters (Heger, Cumming \& Woosley 2007). These authors also found that all the bursts that come up immediately after the $\mathrm{mHz}$ QPOs had positive convexity, suggesting that both phenomena happen at the NS equator.

From the theoretical side, Heger et al. (2007) suggested that the $\mathrm{mHz}$ QPOs are produced by marginally stable nuclear burning of He on the NS surface. The model of Heger et al. (2007) predicts an oscillatory mode of burning, with a period of $\approx 100 \mathrm{~s}$ (similar to what is observed), and only happening near the transition between stable and unstable burning (first predicted by Paczynski 1983), i.e. within a very narrow range of mass accretion rate. However,

${ }^{1}$ Linares et al. (2010) reported $\mathrm{mHz}$ QPOs from the $11 \mathrm{~Hz}$ pulsar IGR J17480-2446, but the properties of the QPOs are quite different compared with those of the listed sources. Ferrigno et al. (2017) found $\mathrm{mHz}$ QPOs in the accreting millisecond X-ray pulsar IGR J00291+5934, but the QPO origin is unclear. while in this model the oscillations appear at accretion rates close to the Eddington rate, observationally the QPOs were observed at an order of magnitude lower $L_{\mathrm{X}}$. Heger et al. (2007) explained the discrepancy by proposing that the accreted material covers only $\sim 10$ percent of the NS surface, so that the local accretion rate (accretion rate per unit area) is effectively close to that of Eddington.

The model of Heger et al. (2007) cannot explain all the phenomenology observed (e.g. mHz QPOs and X-ray bursts occurring at the same time and the frequency drift). To further investigate this issue, Keek, Cyburt \& Heger (2014) studied the possible relation between the $\mathrm{mHz}$ QPOs, the composition of the accreted fuel and the nuclear reaction rates. They found that neither the variation in the composition nor the reaction rate are responsible for the appearance of the $\mathrm{mHz}$ QPOs at the observed accretion rate. However, Keek, Langer \& in't Zand (2009) investigated the effect of NS rotation on the stability of the burning. They found that the combination of a turbulent chemical mixing of the accreted fuel and a higher heat flux from the crust increase the stability of He burning, such that it could explain the observed accretion rate at which the $\mathrm{mHz}$ QPOs take place. Most importantly, Keek et al. (2009) found that the QPO frequency correlates with the heat flux from the crust, so that the frequency drift observed by Altamirano et al. (2008a) could be due to the cooling of the deeper layers of the NS where the burning occurs.

EXO 0748-676 is a transient NS LMXB discovered in outburst during EXOSAT observations in February of 1985 (Parmar et al. 1985). Its X-ray light curve exhibits $\sim 8.3$-min duration eclipses, implying an inclination of $\sim 75^{\circ}-82^{\circ}$ (Parmar et al. 1986). The eclipses allowed to estimate an orbital period of $\sim 3.82 \mathrm{~h}$. The source also shows irregular X-ray dips, mostly in the half orbital cycle prior to the eclipse (Parmar et al. 1986). EXO 0748-676 also presents type I X-ray bursts, a few of which exhibited photospheric radius expansion (PRE). Depending on the assumed fuel composition, the distance estimated using PRE bursts was between $d=5.9 \pm 0.9$ and $7.4 \pm 0.9 \mathrm{kpc}$ (Wolff et al. 2005; Galloway et al. 2008). Homan \& van der Klis (2000) detected a kHz QPO during the persistent emission of an observation of this source with RXTE. Galloway et al. (2010) reported a 552-Hz burst oscillation, associated with the spin frequency of the NS in EXO 0748-676.

\section{OBSERVATIONS AND DATA ANALYSIS}

We used all pointed observations of EXO 0748-676 obtained with the Proportional Counter Array (PCA; for instrument information see Jahoda et al. 2006) aboard the Rossi X-ray Timing Explorer (RXTE). We studied a total of 749 observations taken over nearly 15 yr (March 1996-September 2010). An observation is usually composed of more than an orbit (each one of $\sim 90$ min long duration), separated by data gaps of at least $1 \mathrm{ks}$. In a few occasions the conditions were such that RXTE continuously observed the source for up to $17 \mathrm{ks}$. Thus, our data sets consist of data segments of length $\sim 0.4-17 \mathrm{ks}$. We used $1 \mathrm{~s}$ resolution PCA (observed in Event, Good-Xenon, and Single Bit modes) data to create light curves. Under the assumption that the $\mathrm{mHz}$ QPOs are stronger at lower energies, we followed Altamirano et al. (2008a) and searched for them in the $\approx 2-5 \mathrm{keV}$ energy range (channels 0-10), applying the Lomb-Scargle periodogram (Lomb 1976; Scargle 1982; Press et al. 1992) to each gap-free segment separately. In those segments in which one or more type I X-ray bursts were detected, we searched for periodicities before, after and in between bursts. When an eclipse was detected, we searched for variability before and after it. We found that the oscillations in the $\approx 2-5 \mathrm{keV}$ energy band are evident 


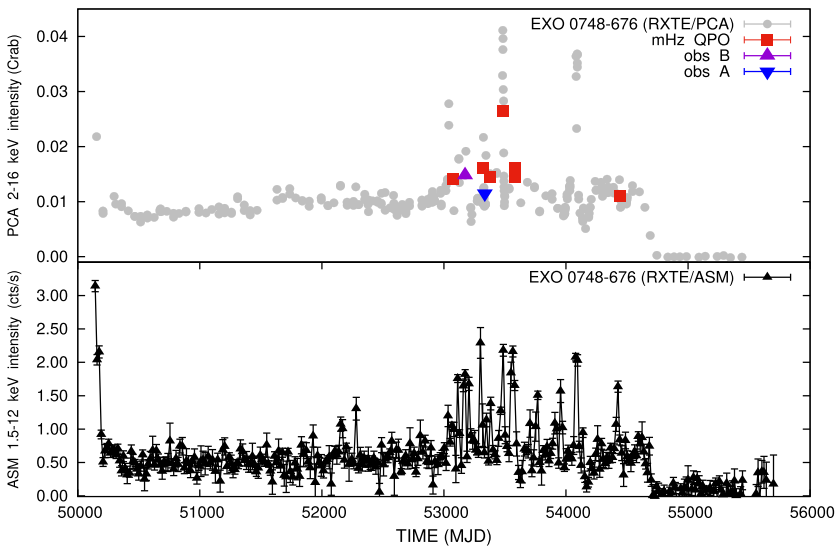

Figure 1. Upper panel: 1996-2010 RXTE/PCA (2-16 keV, open black circles) light curve of EXO 0748-676. Each point corresponds to the average intensity per day when data were available (all type I bursts and eclipses have been removed). The filled red squares represent the dailyaverage count rate of the source when the $\mathrm{mHz}$ QPOs were found. Note that these cases are concentrated mostly in a specific period of time. Note also that the source faded from around MJD 54800. Lower panel: 1996-2010 15-d averaged RXTE/ASM (1.5-12 keV, filled black triangles) light curve of EXO 0748-676. Several flares were detected from MJD 53000, until the source transitioned to the quiescent state (MJD 54800).

from the light curves. In all cases, the detections reported in this Letter are above the $3 \sigma$ level. Following Altamirano et al. (2008a), we calculate the significance level taking into account the number of frequencies searched and assuming white noise as prescribed in the method of Press et al. (1992). We also measured the frequency of the $\mathrm{mHz} \mathrm{QPO}, v_{\mathrm{QPO}}$, as the frequency at which the power reaches its maximum value in its respective periodogram.

In order to create the colour-colour and hardness-intensity diagrams, we calculated colours using the 16-s time-resolution Standard 2 mode data and followed the exact same method used by Altamirano et al. (2008b), with the addition that for this source we identified and excluded eclipsing episodes as well as type I X-ray bursts. We defined the soft colour as the 3.0-6.0 keV/2.0-3.5 keV count rate ratio, the hard colour as the 9.7-16.0 keV/6.0-9.7 keV count rate ratio, and the intensity as the $2.0-16.0 \mathrm{keV}$ count rate. All values were normalized by the colours of the Crab in the same energy bands.

\section{RESULTS}

In Fig. 1 we show both the $1.5-12 \mathrm{keV}$ monitoring 15 -d average light curve from the All-Sky Monitor (ASM; Levine et al. 1996) and the 2-16 keV light curve obtained from RXTE/PCA pointed observations of EXO 0748-676. The intensity of the source was approximately constant, from MJD 50000 to MJD 53000, after which a number of X-ray flares occurred, and EXO 0748-676 transitioned to the quiescent state.

In Fig. 2 we show the colour-colour diagram (CCD) of EXO 0748-676. This plot resembles the CCD of the atoll source $4 \mathrm{U}$ 1636-53 (Altamirano et al. 2008a), even if the softest branch is missing (so-called banana branch). We identified the possible extreme island state (EIS) with the hardest colours (which is the most populated part of the diagram), the island state (IS) with intermediate colours, and the lower left banana (LLB) state with the softest colours. This last identification is supported by the detection of $\mathrm{kHz}$ QPOs (Homan \& van der Klis 2000), which are generally

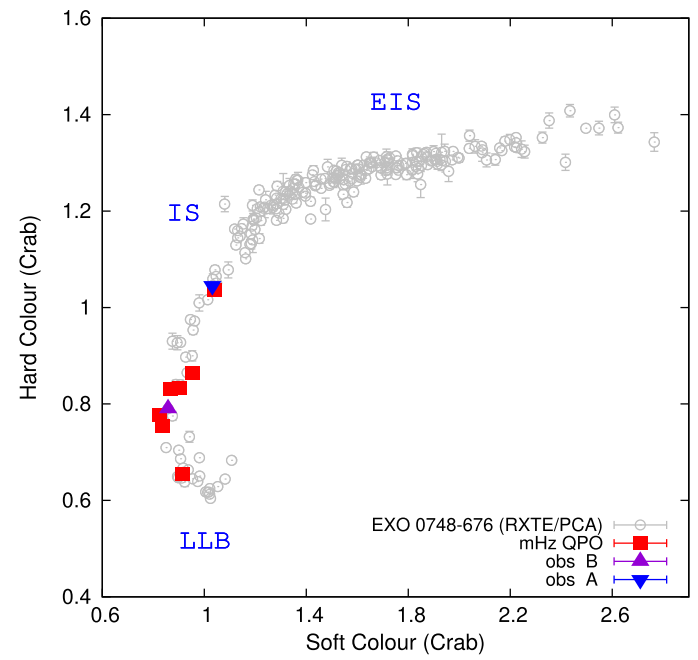

Figure 2. Colour-colour diagram (CCD) of all RXTE observations of EXO 0748-676. Each open black circle represents the daily-average colour of the source from all RXTE observations, excluding the times of bursts, eclipses, and dips. The colours of EXO 0748-676 are normalized to the colours of Crab. The filled red squares represent the colour of the persistent emission of the source when the $\mathrm{mHz}$ QPOs were found. Note also that the source was only observed in certain states: extreme island state (EIS), island state (IS), and lower left banana (LLB).

observed in this state (e.g. review by van der Klis 2000). A reliable identification of the states would require a detailed variability study (e.g. van Straaten et al. 2003, Altamirano et al. 2008b) which is beyond the scope of this Letter.

We detected $\mathrm{mHz}$ QPOs with frequencies between $\sim 5.3$ and $\sim 12.8 \mathrm{mHz}$ in 14 data segments (all shorter than $4 \mathrm{ks}$, distributed in 11 observations) during the flaring period between 2004 March and 2007 December (MJD 53071 and 54439, respectively; see Fig. 1). All detections were found when the source was between $\sim 1$ per cent and $\sim 3$ per cent $L_{\mathrm{Edd}}$, assuming a distance of $7.1 \mathrm{kpc}$ and a $3-50 \mathrm{keV}$ $L_{\text {Edd }}=2.5 \times 10^{38} \mathrm{erg} \mathrm{s}^{-1}$.

The $\mathrm{mHz}$ QPOs (filled red squares; see Fig. 2) appear when the source has hard colour $\lesssim 0.9$ and soft colour $\lesssim 1$, that is, probably the LLB. We also found $\mathrm{mHz}$ QPOs in two segments that fall in an intermediate state, close to the transition between the banana and the island states. In all cases the $2-5 \mathrm{keV}$ fractional rms amplitude of the QPOs was $\sim 4$ per cent, and the QPOs were not significantly detected for energies higher than $\sim 7 \mathrm{keV}$, consistent with the results of Revnivtsev et al. (2001).

In Fig. 3 we show a representative example of a background subtracted light curve of two consecutive observations (obsIDs 80040-01-11-00, -01; from now on, observation 'B'; see Figs 1 and 2). The oscillations can be seen from the beginning of the first observation to $\sim 10.5 \mathrm{ks}$ until an X-ray burst occurred, after which the QPOs disappeared. The QPOs were not detected after the burst for $11.3 \mathrm{k}$ until the end of that observation. The disappearance of the $\mathrm{mHz}$ QPOs after the X-ray burst is consistent with what Revnivtsev et al. (2001) and Altamirano et al. (2008a) found for other sources, and suggests that the physical processes producing the QPOs and the bursts are connected. In the inset of Fig. 3 we show the periodogram of the first data segment. A prominent QPO at $\sim 8 \mathrm{mHz}$ and a possible harmonic at $\sim 16 \mathrm{mHz}$ can be seen.

Altamirano et al. (2008a) found a systematic drift in the frequency of the $\mathrm{mHz}$ QPOs of some observations of the NS LMXB 4U 1636-53. The length of the segments where we detected $\mathrm{mHz}$ QPOs 


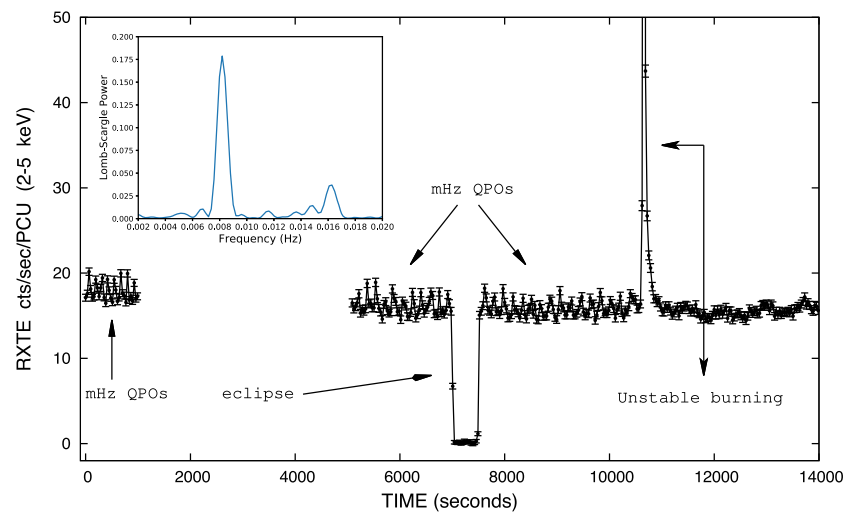

Figure 3. RXTE/PCA light curve $(\approx 2-5 \mathrm{keV})$ of EXO $0748-676$ rebinned to $32 \mathrm{~s}$ in which the $\mathrm{mHz}$ QPOs are detected (obs 'B'; label as in Figs 1 and 2). The oscillations are present in three segments, two before and one after the eclipse. About $10.5 \mathrm{ks}$ from the start of the first observation, a type I X-ray burst occurred and the QPOs, apparent before the burst, disappeared. The inset shows the Lomb-Scargle periodogram of the first $\sim 1200 \mathrm{~s}$ of the data. A significant QPO is present at a frequency of $8 \mathrm{mHz}$, and a second harmonic component at $\sim 16 \mathrm{mHz}$ appears in the plot.

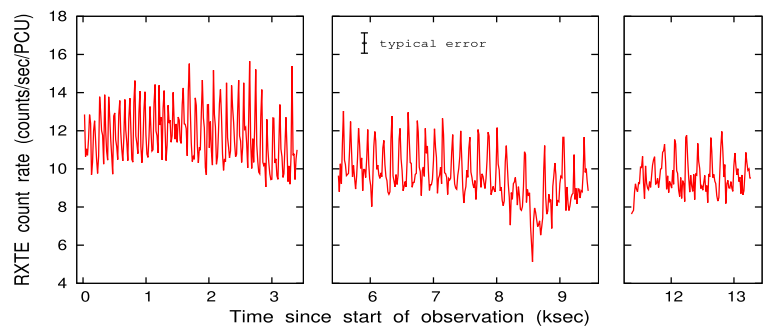

Figure 4. Background corrected light curve $(\approx 2-5 \mathrm{keV})$ of the observation 'A' (label as in Figs 1 and 2) of EXO 0748-676 taken with RXTE/PCA, using 10-s time bins. QPOs are clearly visible through the whole observation.

are generally too short to measure frequency drift; however in two cases we were able to combine data to test for drifts. The first case is shown in Fig. 3. The QPO frequency drifts from segment to segment, starting at $\sim 8.2 \mathrm{mHz}$ (first segment), and drifting to $\sim 5.8$ and $\sim 5.4 \mathrm{mHz}$, before and after the eclipse, respectively. Assuming that the QPOs were always present until the burst onset, the frequency decreased at an average rate of $\sim 0.26 \mathrm{mHz} \mathrm{ks}^{-1}$. The second case is from obsID 90039-01-03-05 (three orbits; from now on, observation 'A'; see Figs 1 and 2), where the QPO frequency drifts from about $\sim 13 \mathrm{mHz}$ to less than $6 \mathrm{mHz}$. Fig. 4 shows the light curve and Fig. 5 the dynamical power spectrum of this observation. The QPOs in this observation persisted for about $13 \mathrm{ks}$ (assuming continuity during data gaps), and their frequency drifted at an average rate of $\sim 0.56$ $\mathrm{mHz} \mathrm{ks}^{-1}$.

EXO 0748-676 was close to the intermediate state when the QPOs exhibited the frequency drifts, consistent with what Altamirano et al. (2008a) found in 4U 1636-53. Finally, excluding those cases where we observed a drift, the frequency of the $\mathrm{mHz}$ QPOs were between $\sim 6.7$ and $\sim 8.7 \mathrm{mHz}$. Our results, therefore, show that EXO 0748-676 is the sixth source that displays this kind of QPO and the second one where a frequency drift is observed.

\section{DISCUSSION}

We report the discovery of $\mathrm{mHz}$ QPOs in the NS LMXB EXO 0748-676. The QPO frequency ranged between $\sim 5$ and $\sim 13 \mathrm{mHz}$,

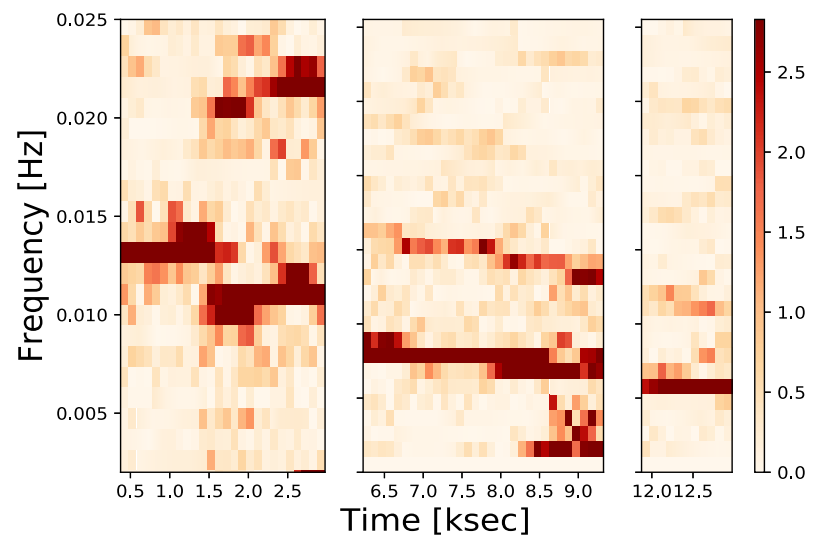

Figure 5. Dynamical power spectrum corresponding to the RXTE observation 'A' (label as in Figs 1 and 2) of EXO 0748-676. The plot, which was made using a $700 \mathrm{~s}$ sliding window with steps of $50 \mathrm{~s}$, shows the evolution of a frequency component associated with the $\mathrm{mHz}$ QPOs, decreasing from $\approx 13 \mathrm{mHz}$ down to $\approx 6 \mathrm{mHz}$.

its amplitude was $\sim 4$ per cent at low energy ( $2-5 \mathrm{keV})$, and in at least two cases the QPO frequency decreased in time. All the detections occurred when the source energy spectrum was relatively soft, as suggested by the CCD. In one case we can confirm that the QPO disappeared after the onset of a thermonuclear X-ray burst (in none of the cases we saw the QPO disappear without the onset of a burst). All these observational properties are consistent with those observed in the accreting NS LMXBs 4U 1636-53, 4U 1608-52, and Aql X-1 (Revnivtsev et al. 2001; Altamirano et al. 2008a), 4U 1323-619 (Strohmayer \& Smith 2011), and GS 1826-238 (Strohmayer et al. 2018, although in this source the amplitude of the $\mathrm{mHz}$ QPO increased with energy), suggesting that we are observing the same phenomenology in six different accreting systems. These $\mathrm{mHz}$ QPOs have been associated with an oscillatory mode of $\mathrm{He}$ burning on the NS surface, generally referred as marginally stable nuclear burning (e.g. Heger et al. 2007; Keek et al. 2009).

Linares et al. (2010) reported the detection of $\mathrm{mHz}$ QPOs with a frequency of $\sim 4.5 \mathrm{mHz}$ during the 2010 outburst of the NS transient IGR J17480-2446. In later work, Linares et al. (2012) reported that these $\mathrm{mHz}$ QPOs appear as the result of a smooth evolution of the thermonuclear bursts properties: as the luminosity of the source increased (decreased), the burst rate increased (decreased), and the fluence of each burst decreased (increased), so that at luminosities higher than $L_{2-50 \mathrm{keV}} \sim 10^{38} \mathrm{erg} \mathrm{s}^{-1}$, bursts appeared in the light curve as a QPO with a period of $\sim 3 \mathrm{~min}$. These results suggested that the burst rate and burst fluence are a function of the luminosity (accretion rate). This behaviour is inconsistent with our findings, where the $\mathrm{mHz}$ QPO and the type I X-ray bursts occur at the same observed luminosity. However, it is currently under debate if the phenomenology observed in IGR J17480-2446 and the six sources listed above are produced by different physical processes, or it is the same physical process under different conditions (see discussion in Linares et al. 2012). For example, different composition of the burning material, different accretion geometry (i.e. accretion mainly on to the NS equator versus spherical accretion on to most of the NS surface), different NS spin frequency and/or magnetic field, and a combination of all these, can affect the burning process and how we detect it.

Ferrigno et al. (2017) detected a QPO at an approximately constant frequency of $\sim 8 \mathrm{mHz}$ in the accreting millisecond $\mathrm{X}$ ray pulsar (AMXP) IGR J00291+5934. The QPO was persistently 
present during an 80-ks XMM-Newton observation. Its rms amplitude strongly decreased with energy, from $\sim 30$ per cent at $\lesssim 1 \mathrm{keV}$ to $\sim 6$ per cent at $\sim 10 \mathrm{keV}$ (the $0.5-11 \mathrm{keV}$ average rms amplitude was $\sim 15$ per cent). Ferrigno et al. (2017) argued that the non-detection of type I X-ray bursts since IGR J00291+5934 discovery (Eckert et al. 2004; Markwardt, Swank \& Strohmayer 2004; Falanga et al. 2005, and also see De Falco et al. 2017), together with the measured properties of the oscillations, strongly suggested that the QPOs they found were not due to marginally stable burning (at least, not in the form of those described in Revnivtsev et al. 2001; Heger et al. 2007; Altamirano et al. 2008a). Ferrigno et al. (2017) instead suggested that the oscillations they found were more similar to those of the socalled 'heartbeats' (and other type of similar variability) observed in the black holes GRS 1915+105 (see e.g. Belloni et al. 2000), IGR J17091-3624 (Altamirano et al. 2011), and potentially the NS system known as the Rapid Burster (Bagnoli \& in't Zand 2015).

\subsection{Frequency drifts and source state}

Altamirano et al. (2008a) found that the $\mathrm{mHz}$ QPOs in 4U 1636-53 would show clear frequency drifts if the system was observed close to the transition between the island and banana states. If the QPOs were detected in the banana state, then the QPO frequency did not drift. Our results on EXO 0748-676 are consistent with those of Altamirano et al. (2008a): in the only two instances in which we observe frequency drifts (A and B, see Fig. 2), EXO 0748-676 was in an intermediate state.

The QPOs in observation 'A' showed a faster frequency drift than we observed in observation ' $\mathrm{B}$ ' $\left(\sim 0.56 \mathrm{mHz} \mathrm{ks}^{-1}\right.$ versus $\sim 0.26 \mathrm{mHz}$ $\mathrm{ks}^{-1}$, respectively). In terms of spectral states, observation ' $\mathrm{A}$ ' is at a harder state than ' $\mathrm{B}$ '. Under the assumption that in the banana state we would not see any frequency drift (as reported by Altamirano et al. 2008a, for $4 \mathrm{U}$ 1636-53), our results appear to suggest that the rate at which the QPO frequency decreases is related to the spectral state, where the rate is maximum at the hardest colours, and decreases as the source evolves to softer states, where we do not expect to see any drift at all. This is not a scenario that can be tested with our RXTE data of EXO 0748-676; however, it could be tested with other sources (e.g. in 4U 1636-53, where Altamirano et al. 2008a reported 22 instances of $\mathrm{mHz}$ QPOs with frequency drifts).

Finally, our results show that the frequency drift is not a unique characteristic of the marginally stable burning in 4U 1636-53, but that can be seen in other sources. This opens a window of opportunity to potentially better understand this type of burning. For example, comparison of the $\mathrm{mHz}$ QPOs characteristics between sources could shed light into the dependence of the QPO frequency drift, and the change in heat flux from the NS crust (Keek et al. 2009).

\section{ACKNOWLEDGEMENTS}

Giulio Cesare Mancuso (GCM) thanks the Royal Society International Exchanges program for their support. Diego Altamirano (DA) acknowledges support from the Royal Society. Jorge Ariel Combi (JAC), Federico Garcia (FG), and GCM were partially supported by Proyectos de Investigacion Plurianuales (PIP) 0102 Consejo Nacional de Investigaciones Cientificas y Tecnicas (CONICET). This work received financial support from Proyectos de Investigacion Cientifica y Tecnologica (PICT)-2017-2865 Agencia Nacional de Promocion Cientifica y Tecnologica (ANPCyT). Lyu is supported by National Natural Science Foundation of China (grant
No.11803025); and Hunan Provincial Natural Science Foundation (grant No. 2018JJ3483).

This research has made use of data and/or software provided by the High Energy Astrophysics Science Archive Research Center (HEASARC), which is a service of the Astrophysics Science Division at National Aeronautics and Space Administration/Goddard Space Flight Center (NASA/GSFC) and the High Energy Astrophysics Division of the Smithsonian Astrophysical Observatory. This research has made use of NASA's Astrophysics Data System.

\section{REFERENCES}

Altamirano D., van der Klis M., Wijnands R., Cumming A., 2008a, ApJ, 673, L35

Altamirano D., van der Klis M., Méndez M., Jonker P. G., Klein-Wolt M., Lewin W. H. G., 2008b, ApJ, 685, 436

Altamirano D. et al., 2011, ApJ, 742, L17

Bagnoli T., in't Zand J. J. M., 2015, MNRAS, 450, L52

Belloni T., Klein-Wolt M., Méndez M., van der Klis M., van Paradijs J., 2000, A\&A, 355, 271

De Falco V., Kuiper L., Bozzo E., Galloway D. K., Poutanen J., Ferrigno C., Stella L., Falanga M., 2017, A\&A, 599, A88

Eckert D., Walter R., Kretschmar P., Mas-Hesse M., Palumbo G. G. C., Roques J.-P., Ubertini P., Winkler C., 2004, Astron. Telegram, 352, 1

Falanga M. et al., 2005, A\&A, 444, 15

Ferrigno C. et al., 2017, MNRAS, 466, 3450

Galloway D. K., Muno M. P., Hartman J. M., Psaltis D., Chakrabarty D., 2008, ApJS, 179, 360

Galloway D. K., Lin J., Chakrabarty D., Hartman J. M., 2010, ApJ, 711, L148

Hasinger G., van der Klis M., 1989, A\&A, 225, 79

Heger A., Cumming A., Woosley S. E., 2007, ApJ, 665, 1311

Homan J., van der Klis M., 2000, ApJ, 539, 847

Homan J., Wijnands R., van den Berg M., 2003, A\&A, 412, 799

Jahoda K., Markwardt C. B., Radeva Y., Rots A. H., Stark M. J., Swank J.

H., Strohmayer T. E., Zhang W., 2006, ApJS, 163, 401

Jonker P. G., van der Klis M., Wijnands R., 1999, ApJ, 511, L41

Jonker P. G., Méndez M., van der Klis M., 2000, ApJ, 540, L29

Keek L., Langer N., in't Zand J. J. M., 2009, A\&A, 502, 871

Keek L., Cyburt R. H., Heger A., 2014, ApJ, 787, 101

Levine A. M., Bradt H., Cui W., Jernigan J. G., Morgan E. H., Remillard R., Shirey R. E., Smith D. A., 1996, ApJ, 469, L33

Linares M. et al., 2010, Astron. Telegram, 2958, 1

Linares M., Altamirano D., Chakrabarty D., Cumming A., Keek L., 2012, ApJ, 748, 82

Lomb N. R., 1976, Ap\&SS, 39, 447

Lyu M., Méndez M., Altamirano D., 2014, MNRAS, 445, 3659

Lyu M., Méndez M., Zhang G., Keek L., 2015, MNRAS, 454, 541

Lyu M., Méndez M., Altamirano D., Zhang G., 2016, MNRAS, 463, 2358

Markwardt C. B., Swank J. H., Strohmayer T. E., 2004, Astron. Telegram, 353,1

Méndez M., van der Klis M., Ford E. C., Wijnands R., van Paradijs J., 1999, ApJ, 511, L49

Paczynski B., 1983, ApJ, 264, 282

Parmar A. N., White N. E., Giommi P., Haberl F., Pedersen H., Mayor M., 1985, IAU Circ., 4039, 1

Parmar A. N., White N. E., Giommi P., Gottwald M., 1986, ApJ, 308, 199

Press W. H., Teukolsky S. A., Vetterling W. T., Flannery B. P., 1992 Numerical Recipes in FORTRAN. The Art of Scientific Computing. 2nd ed., University Press, Cambridge

Pringle J. E., Rees M. J., 1972, A\&A, 21, 1

Psaltis D., Chakrabarty D., 1999, ApJ, 521, 332

Revnivtsev M., Churazov E., Gilfanov M., Sunyaev R., 2001, A\&A, 372, 138

Scargle J. D., 1982, ApJ, 263, 835

Strohmayer T., Bildsten L., 2006, New Views of Thermonuclear Bursts, Compact Stellar X-ray Sources 39. Cambridge University Press, p. 113 
Strohmayer T. E., Smith E. A., 2011, Astron. Telegram, 3258, 1

Strohmayer T. E. et al., 2018, ApJ, 865, 63

van der Klis M., 2000, ARA\&A, 38, 717

van der Klis M., 2006, Rapid X-ray Variability. Compact Stellar X-ray Sources. Cambridge University Press, p. 39

van Straaten S., van der Klis M., Méndez M., 2003, ApJ, 596, 1155

Wijnands R., 2004, Nucl. Phys. B, 132, 496
Wolff M. T., Becker P. A., Ray P. S., Wood K. S., 2005, ApJ, 632, 1099

Yu W., van der Klis M., 2002, ApJ, 567, L67

This paper has been typeset from a $\mathrm{T}_{\mathrm{E}} \mathrm{X} / \mathrm{L} \mathrm{T}_{\mathrm{E}} \mathrm{X}$ file prepared by the author. 